استخدام اوكسيد الاسبيرسيوم (Dy2 $\left.\mathbf{O}_{3}\right)$ كحاجز حراري في عملية طلاء نوع من الفولاذ المقاوم للصدأ

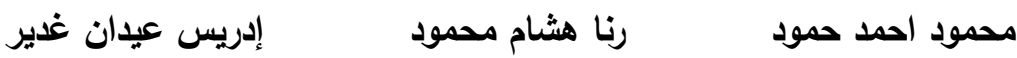

$$
\begin{aligned}
& \text { قسم الفيزياء/ كلية العلوم/ جامعة الموصل }
\end{aligned}
$$$$
\text { (أُنظلم 2012/9/13/ }
$$

\begin{abstract}
الملخص
إن الظروف الصعبة التي تعاني منها بعض المواد نتيجة ظروف عملها استوجب طلاؤها لحمايتها وزيادة أعمارها في الخدمة

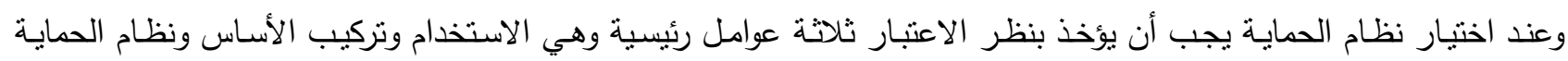
نفسه. وفي هذه الدراسة نم اختبار أنواع معينة من الطلاء الانتشاري بطريقة السمنتة (الطلاء الالوميني المنفرد، الطلاء بالحاجز

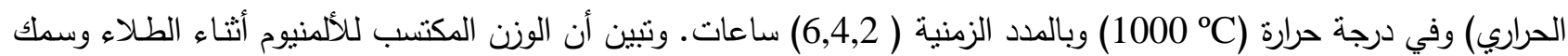
الطلاء يخضع لقانون القطع المكافئ لكلا النوعين من الطلاءات الأمر الذي يؤكد إن عملية الطلاء تكون محكومة بعملية الانتشار وان معدلاتهما تزداد مع زيادة درجات الحرارة. أما الاختبار فقد نم في منصهر كلوريد الصوديوم في درجة (Co

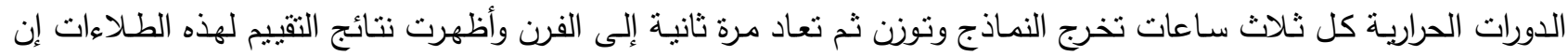
الطلاء المنفرد قد وفر حماية لمدة جيدة للمعدن قيد البحث في حين إن النموذج القياسي غير المطلي قد انهار خلال الدورات

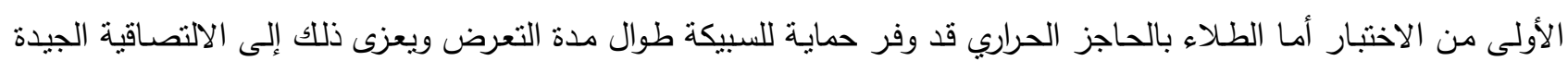

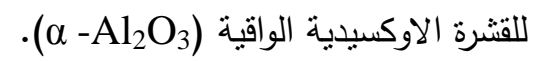

الكلمات الدالة: الطلاء بالحواجز الحرارية، الطلاء الانتشاري، الألمنة التقليدية، التآكل الحار ، سبائك الحديد، حماية المعادن.

\title{
The Use of $\mathrm{Dy}_{2} \mathrm{O}_{3}$ as Thermal Barrier in Aluminide Coating on One Type of Stainless Steel
}

\author{
Mahmood A. Hamood Rana H. Mahmood Edrees E. Kidder \\ Department of Physics/College of Science/ University of Mosul
}

\begin{abstract}
The working hard conducts that some material suffer from lead us to protect it from corrosion and give long life. When we select protective system we shall take three points, the work place, base structure and the protective system it self. In this study, we test two types of diffusion coated by cementation (Single Aluminized Coating, Thermal Barrier Coating) at $\left(1000{ }^{\circ} \mathrm{C}\right)$ with $(2,4,6 \mathrm{~h})$. The up take of aluminum and coating thickness obeys the parabola rule so that the coating process obeys diffusion process and the weight gain rate increase with the temperature increased. The other objective of this study was to see the degradation behavior of the systems during thermal cycling (three hour cycles in enhanced hot - salt solution). The single aluminized has good protects while the uncoated sample failed after five cycles. The thermal barrier coating has an excellent protection during the test time because of the good adhesion of $\mathrm{Al}_{2} \mathrm{O}_{3}$ layer.
\end{abstract}

Keywords: Thermal Barrier Coating, Diffusion Coating, Iron Alloy Aluminizing, Hot Corrosion. 


\section{(المقدمة}

يعذّ الصلب الأوستتايتي أكثر أنواع الصلب استعمالاً نظراً لما يمثاز به من خواص ميكانيكية ومقاومة تآكل جيدنين، لذا يكثر استعماله في الصناعات البتروكيماوية وفي التطبيقات التي تتطلب درجات حرارة عالية وعدد من أجزاء المحركات التوربينية

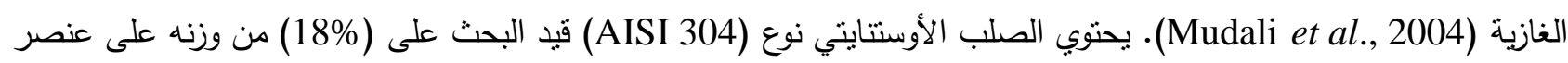

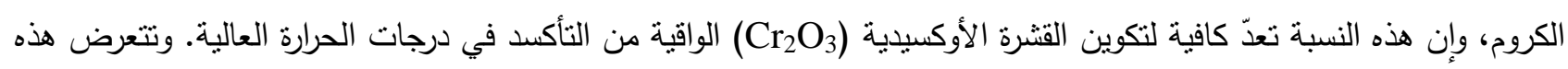
القشرات إلى التساقط أثناء الدورات الحرارية بسبب اختلاف معامل التمدد الحراري مع معامل تمدد الأساس، ويستمر تكوين هذه القشرة على سطح السبيكة بعملية الاندمال (Healing) مما يؤدي إلى استنفاذ عنصر الكروم من السبيكة. تحتوي هذه السبيكة على نسبة قليلة من الكاربون (0.03\%) تؤدي إلى تكوين كاربيدات الكروم التي تترسب عند الحدود الحبيية فضلاً عن تكوينها مركبات كلوريدية $\left(\mathrm{Cr}_{2} \mathrm{Cl}_{3}\right)$ عند استخدامها في الأوساط الحاوية على الكلور ومركباته وهذه المركبات تعمل على زيادة أخرى في تلكي استتفاذ عنصر الكروم مما يؤدي إلى تتاقص نسبته المئوية ومن ثم انهيار السبيكة كلياً.

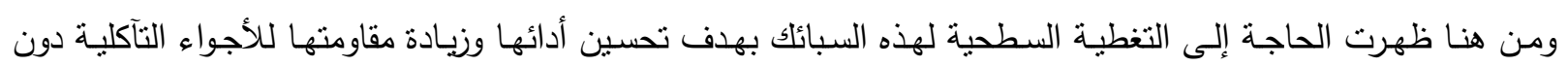
الحاجة إلى إجراء تغييرات في تصميم السبائك وتعقيداتها الصناعية. وبالاعتماد على هذه العوامل، فان القشرة المتكونة على السبيكة قد تنكون:

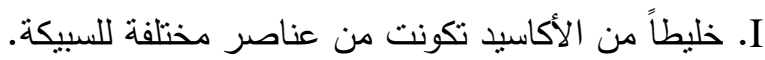

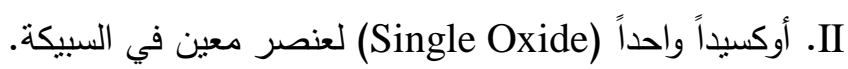
أوكسيداً واحداً أو أكاسيد عديدة على شكل طبقة خارجية مع أكاسيد داخلية لعناصر محددة. III فالحالة الأولى هي حالة التأكسد العام، والتي تتكون في بداية مرحلة التأكسد وتعرف بالأكسدة الوقتية أو العابرة ( Transient

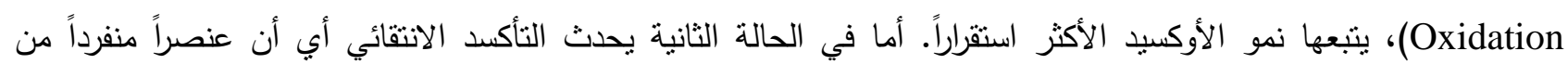
عناصر السبيكة فقط سوف يتأكسد. أما في الحالة الثالثة، وهي الأكثر شيوعاً والأكثر أهمية، فبسبب الفعالية العالية لعناصر محددة، والانتثار الداخلي

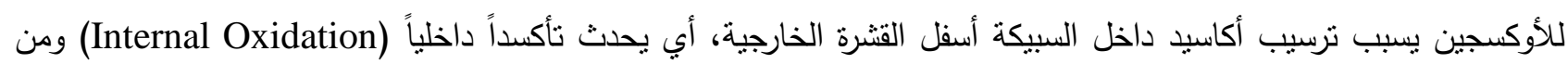
الجدير بالذكر أن تكون الاوكسيد الداخلي يمكن أن يكون مفيداً في بعض الأحيان، فهو بمكن أن يقوي (Strengthen) السبيكة

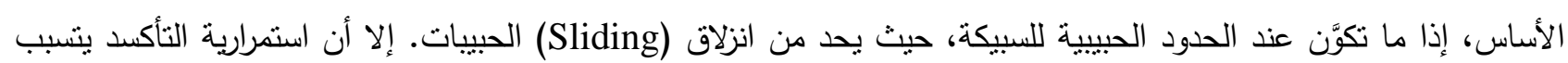
في تأكسد الحبيبات نفسها ومن ثم انهيارها، (Khana, 2004).

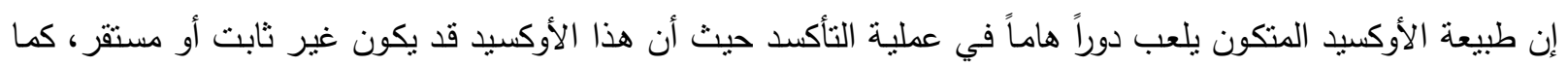

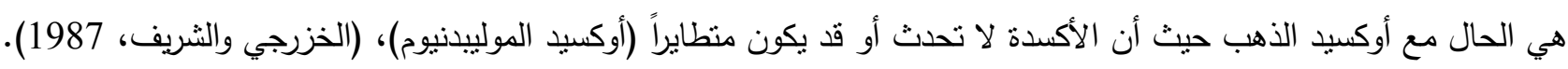

\section{Mechanism of Hot Corrosion}

آلية التآكل الحار

ان التآكل الحار يعد نوعا من أنواع التعرية التي تحصل للسبائك المعدنية نتيجة لترسبات الملوثات منل أملاح كبريتات

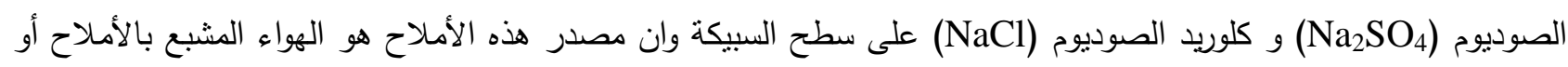
تكون الكبريتات أثناء احتراق الوقود وتعتمد عملية التآكل الحار بشكل كبير جداً على درجة الحرارة حيث يزداد معدل التآكل بزيادة

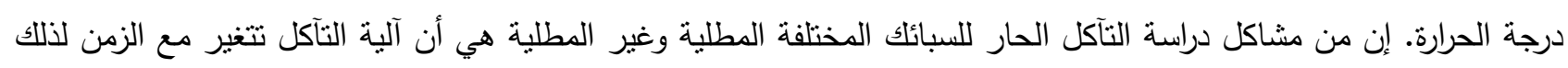

فان عوامل التآكل الحار تؤخذ كدالة للزمن (Khana, 2004).

إن دراسة التآكل الحار في منصهر كلوريد الصوديوم (NaCl) يتضمن دراستين، فإما أن تكون دراسة التآكل في درجات حرارية اقل من درجة انصهار ملح كلوريد الصوديوم (810º C أو أعلى من درجة الانصهار • وقد أشنارت عدة أبحاث إلى تكون 
كرومات الصوديوم Oxychloridation نتيجة تفاعل الكروم أو اوكسيد الكروم مع ملح كلوريد الصوديوم

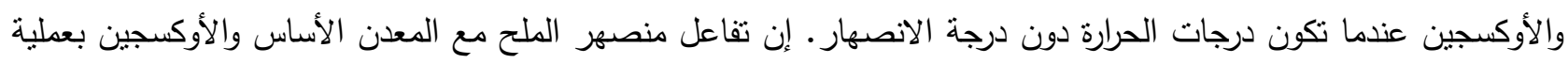

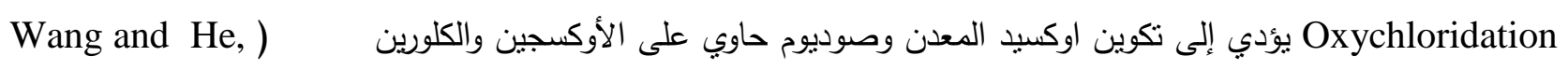
2002)، وهناللك ثلاث اتجاهات لتفاعل الكلورين وهي: 1 - بتحرر الى الغلاف الجوي.

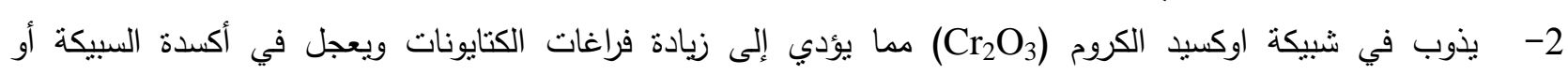
المعدن.

3- يتفاعل مع الأساس مكونا كلوريد المعدن الأساس. وهي مركبات وسطية لتفاعلات chloridatio / oxidation. حسب قوانين الثزموداينمك فان الكروم يمنلك ألفة عالية للتفاعل مع الكلورين لتكوين كلوريد المعدن بينما التكات النيكل يكون خاملا في هذا الاتجاه وييقى في السبيكة الأساس وان عملية تكوين الكوريدات يؤدي إلى هجرة هذه العناصر الى الخارج مخلفة

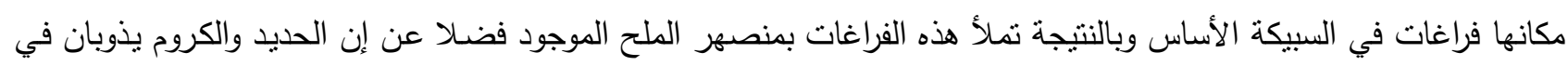

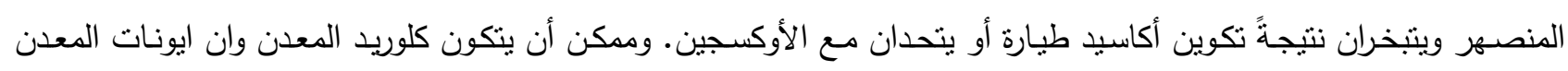
non-protective تذوب في منصهر الملح ومن ثم تتبخر أو تتنتر إلى الخارج ونتفاعل مع الأوكسجين مكونة طبقة غير والقينة duplex-layer scales

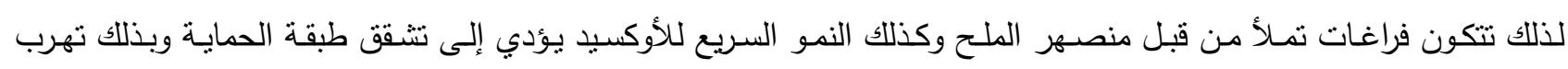

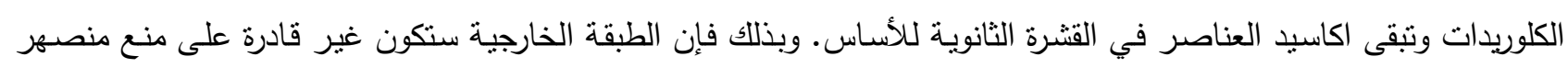

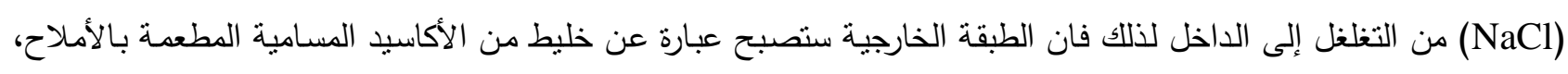

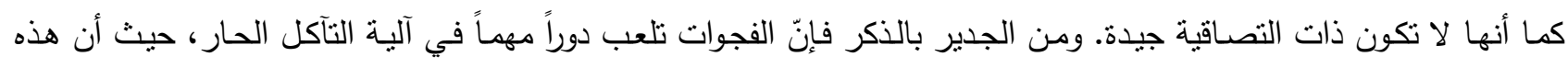

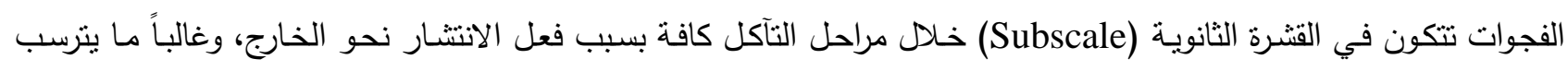
منصهر الملح في هذه الفجوات عند السطح البيني (القشرة-المعدن)، وبفعل درجة الحرارة والزمن فان محتوى هذه الفجوات سيتغلغل Seifedine, ) (Higginson et al., 2006) إلى الداخل مما يؤدي إلى انهيار الطبقة الحامية ومن ثم فنشل السبيكة. $(2008$

\section{Pack Cementation}

تعدّ هذه الطريقة من أوسع طرائق الطلاء الانتشاري استخداماً لما تتميز به من مميزات، فهي من الطرائق الفعالة وغير

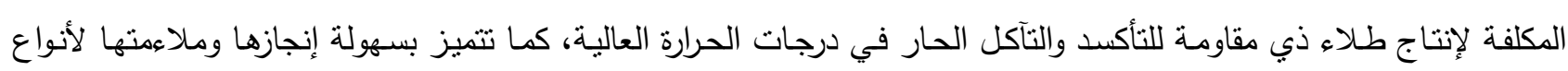
مختلفة من السبائك مثل (Fe- , Ni- , Co-Based)، كما ان الطلاء بهذه الطريقة يكون منتظماً ومتجانساً في السمك والتركيب،

.(Houngninou et al., 2003)

ففي هذه الطريقة يتم غمر الجزء المراد طلاؤه في مزيج من معدن الطلاء (المنيوم، نيكل، كروم) والمادة المنشطة وهي إحدى

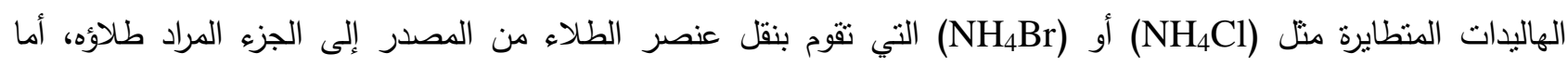
فهي تمنع تكتل المزيج حيث يوضع المزيج في بودقة مصنوعة من الألومينا ويتم إغلاقها من الطرفين وتوضع في فرن

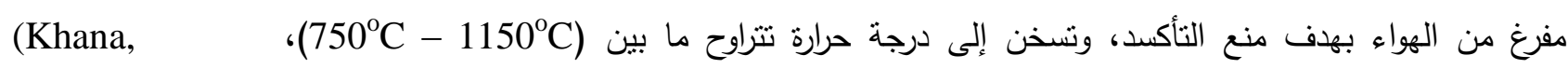

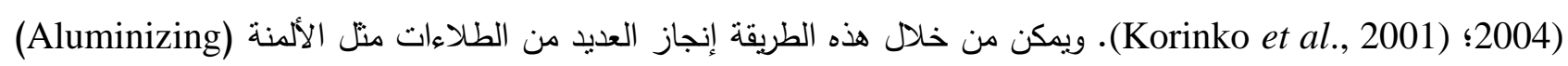

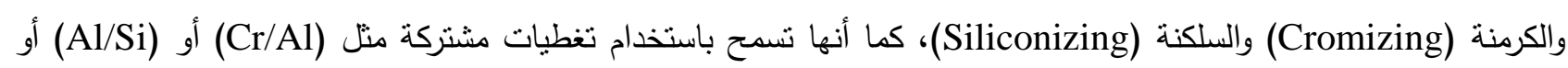
.(Squillace et al., 1999) ؛(Cr/Al/Si) 
ومن العوامل المؤثرة في نوعية الأطوار المتكونة ومواصفات طبقة الطلاء المنجزة بهذه الطريقة هي العوامل الآتية،

:(Levin et al., 1998)

1. مكونات المزيج المحضر .

2. تركيب السبيكة الأساس.

3. 3 درجة الحرارة.

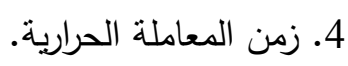

إن استخدام الألمنيوم في الطلاء بطريقة السمنتة يدعى بالألمنة وتعني إنثباع سطح المنتوج بالألمنيوم عن طريق انتشار

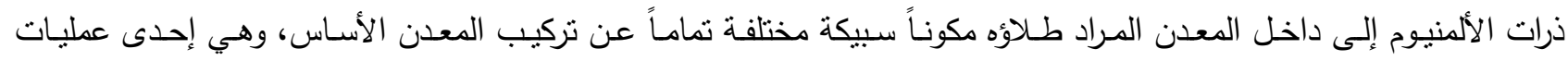
الترسيب الكيميائي بالبخار (CVD) فالتفاعلات الكيميائية التي تحدث خلال هذه العملية هي مشابهة لتلك الموجودة في الـ (CVD) ولكنها تختلف في أنها تجري داخل بودقة الطلاء وكذلك يكون مدى انتقال بخار معدن الطلاء قصيراً جداً من المسحوق إلى الجزء المراد طلاؤه (Tatsai and Ehuang, 2000).

Mechanism of Pack Cementation آلية عملية السمنته إن آليـة السمنتة تكون منشـابهة لكل معادن الطسلاء سواءً استخدم الألمنيوم أو الكروم أم السليكون أو غيرها من معادن الطلاء. وتتضمن عملية الانتشار هذه حدوث الآليتين الآتيتين:

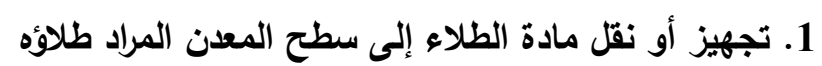
تتم عملية نقل مادة الطلاء إلى سطح المعدن المراد طلاؤه خلا الطو الطور الغازي، ويشترط أن تمنلاك حاملات معدن الطلاء التي هي إحدى الهاليدات المنطايرة ضغطاً بخارياً أعلى من ضغط المعدن المراد طلاؤه (Shahror, 1994)، ويكون التفاعل مع التع سطح السبيكة ترسيب الألمنيوم بالثكل الآتي، (Squillace et al., 1999): $\mathrm{AlXn}+\mathrm{Fe} \rightarrow \mathrm{AlFe}_{\mathrm{Y}}+\mathrm{AlX}_{3}$

$$
\text { حيث إنّ (1/3 }
$$

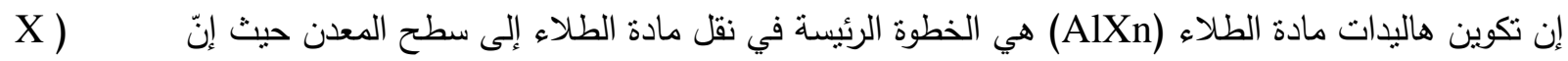

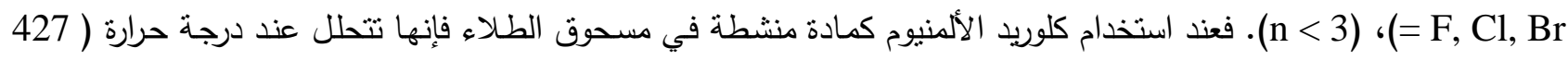
نتيجة النسخين مكونة غاز الأمونيا (NH3)

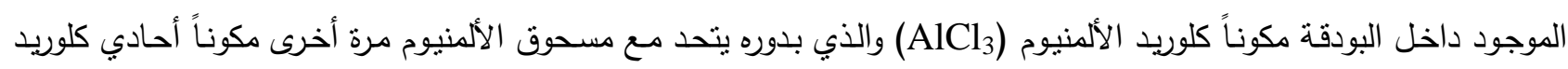
الألمنيوم (Monochloride) أي (AlCl)، فقد وجد أن هذا الهاليد يمثل الوسط الذي ينتقل خلاله الألمنيوم إلى سطح السبيكة

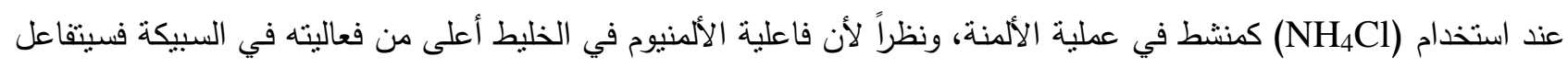

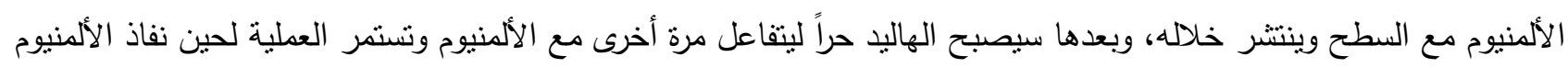
من الخليط. (Khana, 2004).

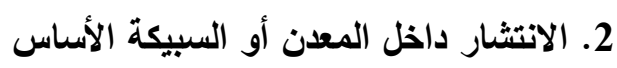
هناك عمليتان رئيستان تحدثان عند سطح السبيكة، الأولى انتشـار الألمنيوم إلى داخل السبيكة الأسـاس، والثانيـة هي الأسياسي انتتار عنصر أو عناصر السبيكة الأساس إلى الطبقة السطحية. وكلتا هاتين العمليتين محكومتان بطاقة تكوين المركبات وانحدار

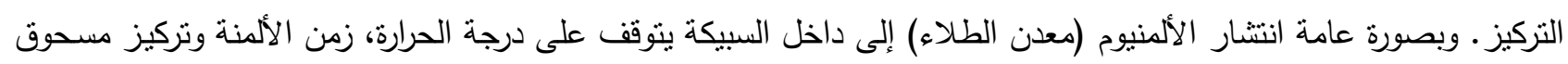
الألمنيوم في خليط الطلاء. 
1 ـ سبيكة من الصلب المقاوم للصدأ (AISI- 304 Stainless Steel) تحتوي على النسب الوزنية الموضحة بالجدول (1). وقد

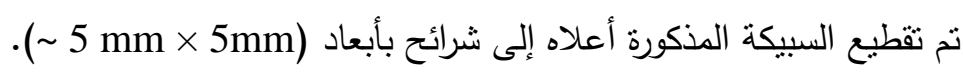

الجدول 1: تركيب سبيكة الصلب المقاوم للصدأ (AISI 304)

\begin{tabular}{|c|c|c|c|c|c|c|c|c|c|}
\hline Element & $\mathbf{C}$ & $\mathbf{M n}$ & $\mathbf{S i}$ & $\mathbf{P}$ & $\mathbf{S}$ & $\mathbf{C r}$ & $\mathbf{N}$ & $\mathbf{N i}$ & $\mathbf{F e}$ \\
\hline Wt. \% & 0.03 & 2.0 & 0.75 & 0.045 & 0.03 & 18 & 0.1 & 8 & Base \\
\hline
\end{tabular}

2. مـزيج مـن مسـحوق الألمنيوم النقي Al ومسـحوق كلوريـ الأمونيوم (NH4Cl (N) كمـادة منشطة وأوكسيد الألمنيوم النقي

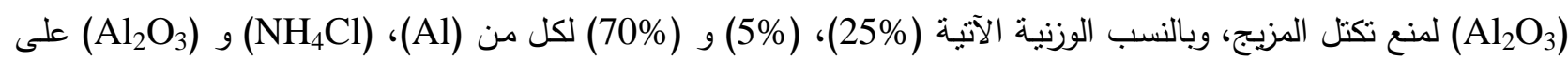

$$
\begin{aligned}
& \text { التوالي. } \\
& \text { 3. أنبوب معدني مقاوم للحرارة مفتوح من أحد الطرفين. } \\
& \text { 4. مضخة تفريغ Sotary Pump. }
\end{aligned}
$$

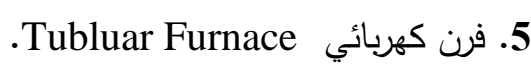

6. Tسبطر لدرجة الحرارة Temperature Controller.

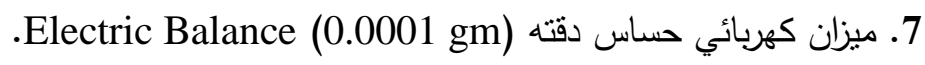

8. جهاز حيود الأشعة السينية

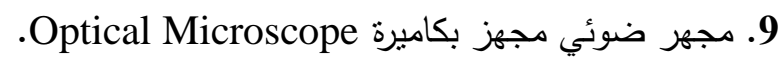

\section{Sample Preparation for Coating}

تهيئة العينات للطلاء

تعد عملية تهيئة العينات للطلاء من الوسائل الضرورية المهمة جداً لنجاح عملية الطلاء. فبعد عملية تقطيع السبيكة نم إجراء عملية التتعيم (Grinding) والصقل (Polishing) للعينات لإزالة الأكاسيد الموجودة على السطح باستخدام ورق التتعيم

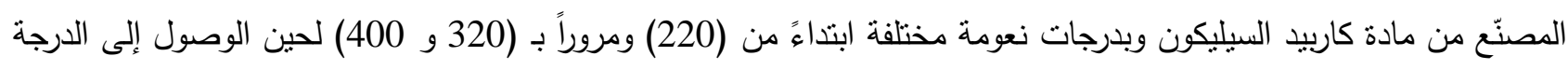

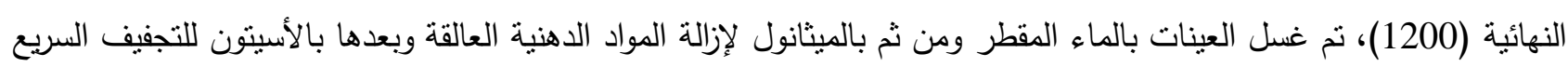
ثم بالماء المقطر .

\section{Single Stage Aluminide Coating}

الطلاء المنفرد بالألمنيوم باءه

تمت عملية الطلاء بالألمنيوم بمرحلة واحدة بطريقة السمنتة (Pack Cementation) التي تعتمد أساساً على عمليات

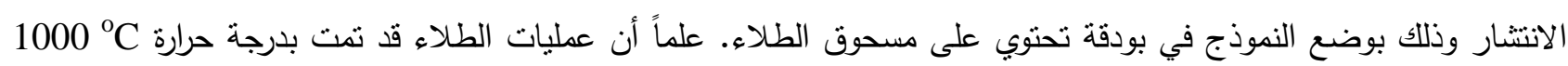
وبالمُدد الزمنية ( 6، 4، 2 ) ساعة وكالآتي.

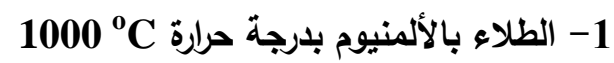

إن الألمنيوم المكتسب (Uptake) الناتج من عملية الألمنة يزداد مع الزمن وفقاً لعلاقة القطع المكافئ وكما في الثكل (1)

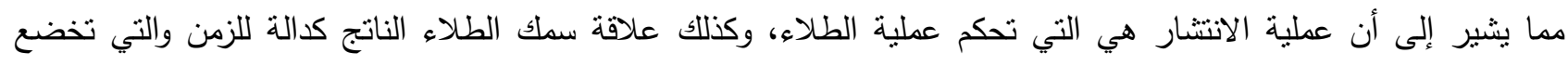
لقانون القطع المكافئ أيضاً. ويضم الجدول (2) معدلات الوزن المكتسب ومعدلات السمك الناتج من الطلاء للمُدد الزمنية

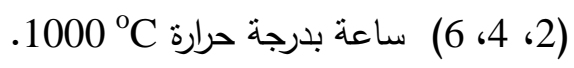




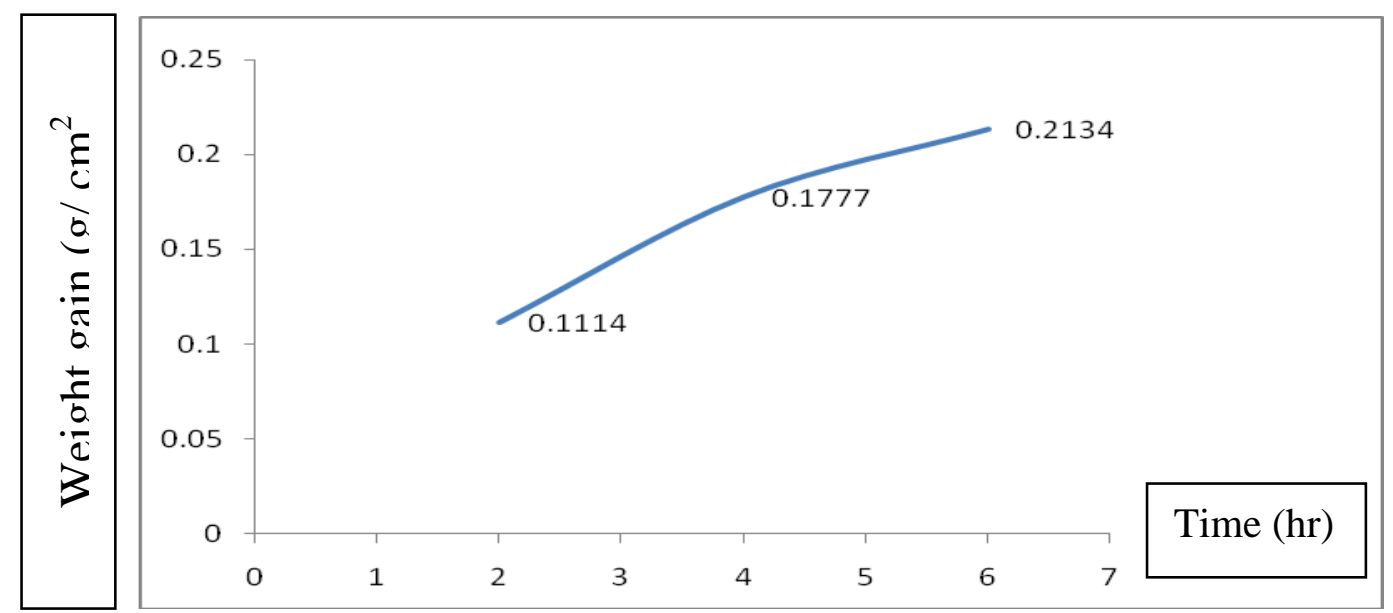

الشكل 1: وزن الألمنيوم المكتسب كدالة للزمن للنماذج المؤلمنة فقط

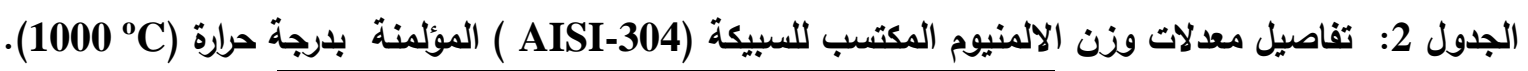

\begin{tabular}{|c|c|l|}
\hline \multirow{2}{*}{ Temp. $^{\mathbf{~}} \mathbf{C}$} & $\begin{array}{c}\text { Coating } \\
\text { Time (h) }\end{array}$ & $\begin{array}{c}\text { Av. Weight } \\
\text { gain } \\
\text { (gm/.cm }\end{array}$ \\
\hline \multirow{3}{*}{1000} & 2 & 0.1114 \\
\cline { 2 - 3 } & 4 & 0.1777 \\
\cline { 2 - 3 } & 6 & 0.2134 \\
\hline
\end{tabular}

وبما أن السبيكة المستخدمة قبد البحث هي ذات أساس حديد فإن طلاءها بالألمنيوم يؤدي إلى تكوين أحد أطوار المركبات الوسطية (حديد - ألمنيوم)، وهذا ما قد حصل فعلاً فقد أظهرت نتائج تحليل حيود الأثتعة السينية XRD الثنكل (2) تعقيدات كثيرة من حيث عدد الأطوار الناتجة ولكنها أكدت تكوين الطور الغني بالألمنيوم (

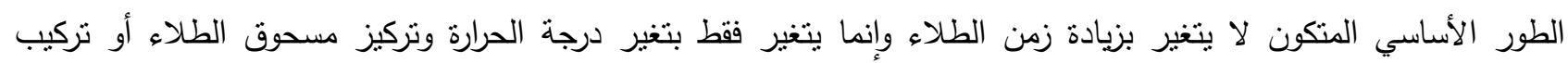
السبيكة الأساس، وهذا ما أكده الباحثون (Morsi et al., 2001) ؛ (Chen et al., 2003).

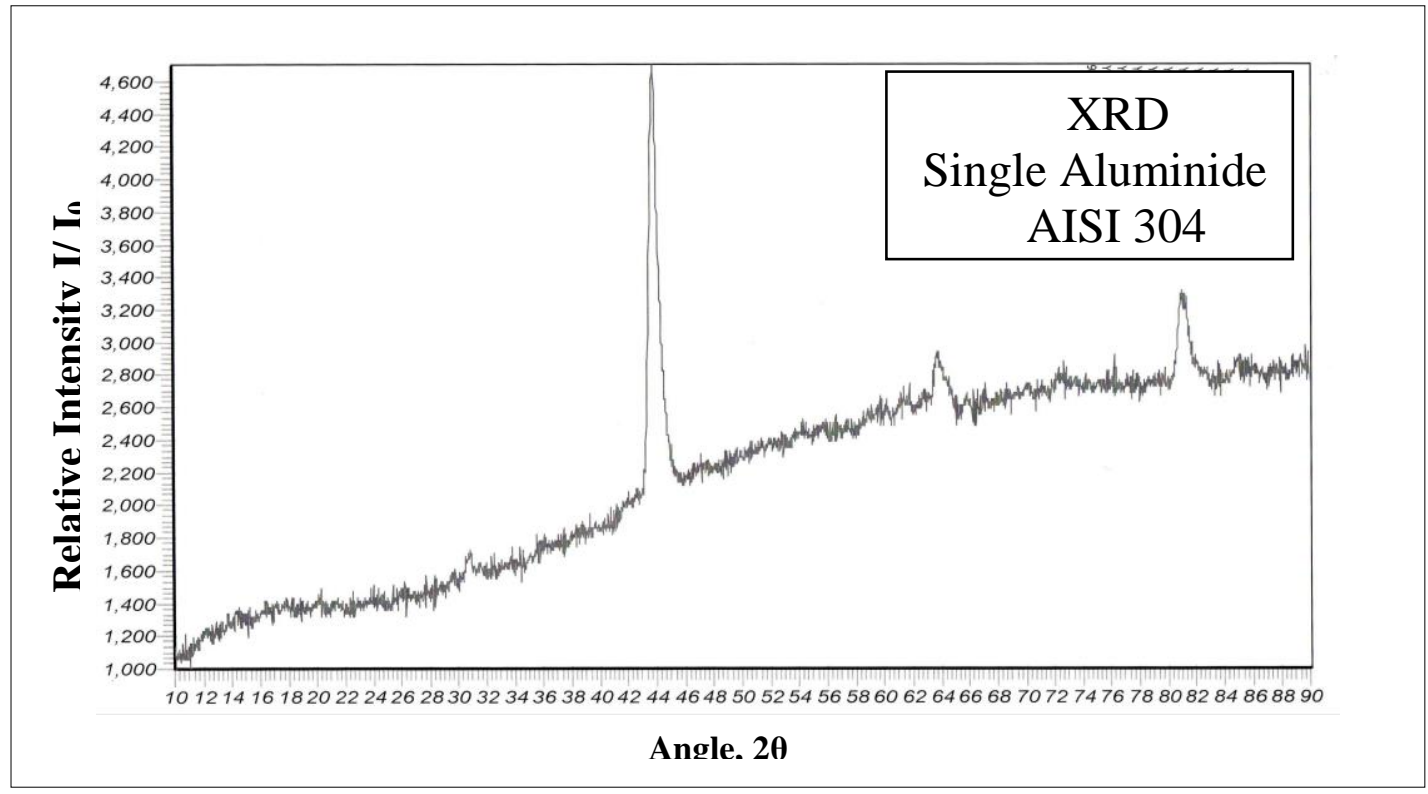

الثكل 2: مخطط حيود الأشعة السينية للنماذج المؤلمنة في درجة حراة (Co0 C 1000) لمدة ساعتين 
ويوضح الجدول (3) نتائج تحليل حيود الأشعة السينية للعينة المطلية لمدة نموذج لهذا النوع من الطلاء، إذ تبين من نموذج

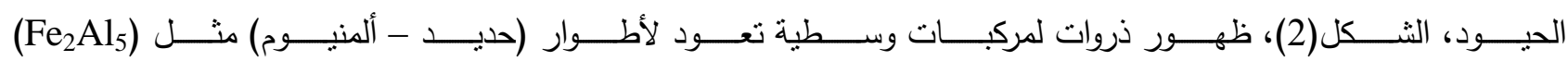

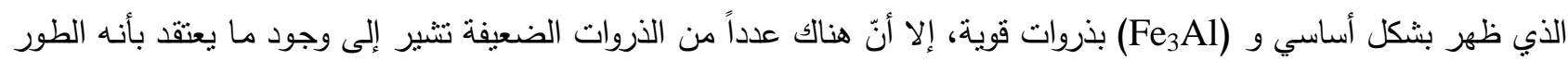

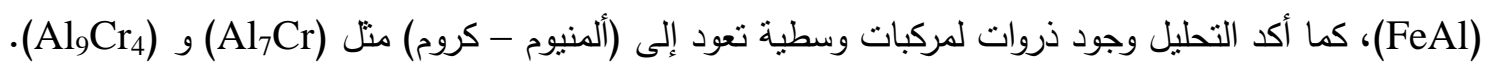

الجدول 3: نتائج فحص الأثعة السينية (XRD) للعينة المطلية بلرجة حرارة Co0 1000 لمدة ساعتين

\begin{tabular}{|c|c|}
\hline نوع الطور & الثدة النسبية \\
\hline $\mathrm{Fe}_{2} \mathrm{Al}_{5}$ & $\mathrm{Vs}$ \\
\hline $\mathrm{Fe}_{3} \mathrm{Al}$ & $\mathrm{S}$ \\
\hline $\mathrm{FeAl}$ & $\mathrm{W}=$ weak \\
\hline $\begin{array}{c}\mathrm{Vs}=\text { very strong } \\
\mathrm{S}=\text { strong }\end{array}$ & \\
\hline
\end{tabular}

أشنارت نتائج الأشعة السينية الثكل (3) إلى أن المنطقة الخارجية قد تكونت بفعل الانتشار الداخلي للألمنيوم مكونة المحلول

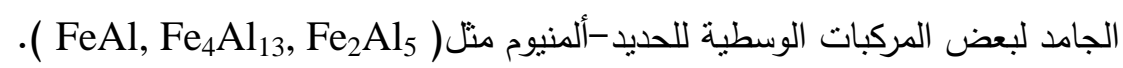
يبدو من ذلك إن المنطقة الداخلية قد تكونت بفعل مساهمة الانتتار الخارجي للحديد بعد تتاقص فعالية الألمنيوم وتكوين الطور ( Fe (الذي أثنارت إلى وجوده نتائج الأتشعة السينية بذروات اقل شدة، ومن اجل استيضاح حدوث هذه الآلية فقد نم دراسة آلية الطلاء حيث تثير إلى زيادة الوزن المكتسب للألمنيوم مع الزمن خلال عملية الطلاء وفقا لعلاقة القطع المكافئ الثكل (1). إن استتزاف الحديد من هذه المنطقة أدى إلى ترسب مركبات العناصر الأخرى للسبيكة والتي تكون قابلية ذوبانها محدودة مثل

1000C اختبار طلاءات الألمنيوم المنفردة المحضرة بدرجة حرارة Testing of Single Aluminide Coatings Prepared at $1000^{\circ} \mathrm{C}$

أن الطلاءات الناتجة بالمُدَد الزمنية (4، 2) ساعة في درجة $1000^{\circ} \mathrm{Ce} \mathrm{Al}_{5}$ (تعتمد على أطوار الحديد الغنية بالألمنيوم

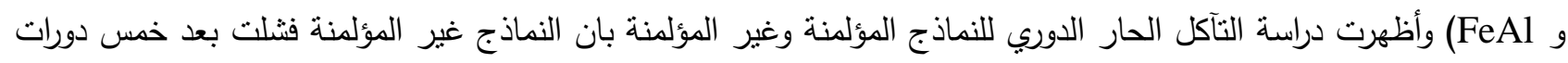
نتيجة نساقط القشرة الأوكسيدية بعد كل دورة من الدورات نتيجة نكون الأوكسيد الطيار (المتبخر) (Voltile Oxide) (Cr3

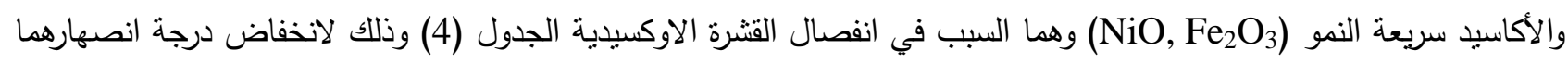
أما النماذج المؤلمنة فقط فقد أظهر الطلاء حماية جيدة للمعدن من التآكل الحار وكما هو مبين من منحني تغير (الوزن - الزمن)

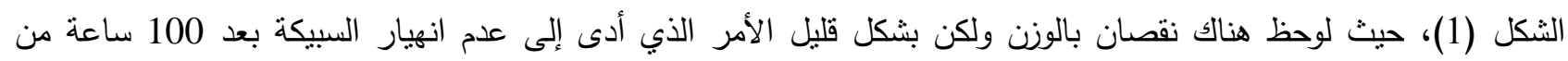
الاختبار القاسي في منصهر كلوريد الاصوديوم بسبب ان القشرة الاوكسيدية الواقية بالتصاقية مقبولة وهي المسؤولة عن توفير الحماية للطلاء وان تساقطها بشكل محدود من بعض مناطق السطح الخارجي لم يؤدي

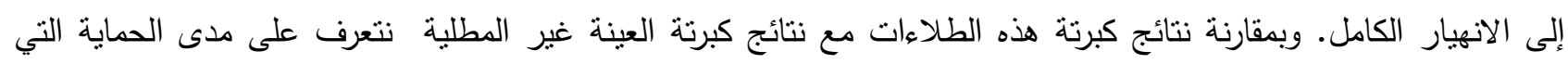
وفرتها هذه الطلاءات للسبيكة كما هو مبين في الثكل (3). أما النماذج المؤلمنة بوجود الحاجز الحراري فقد أثنتت كفاءة عاءته عالية جدا بسبب عدم تأنرها بهذا الاختبار . 


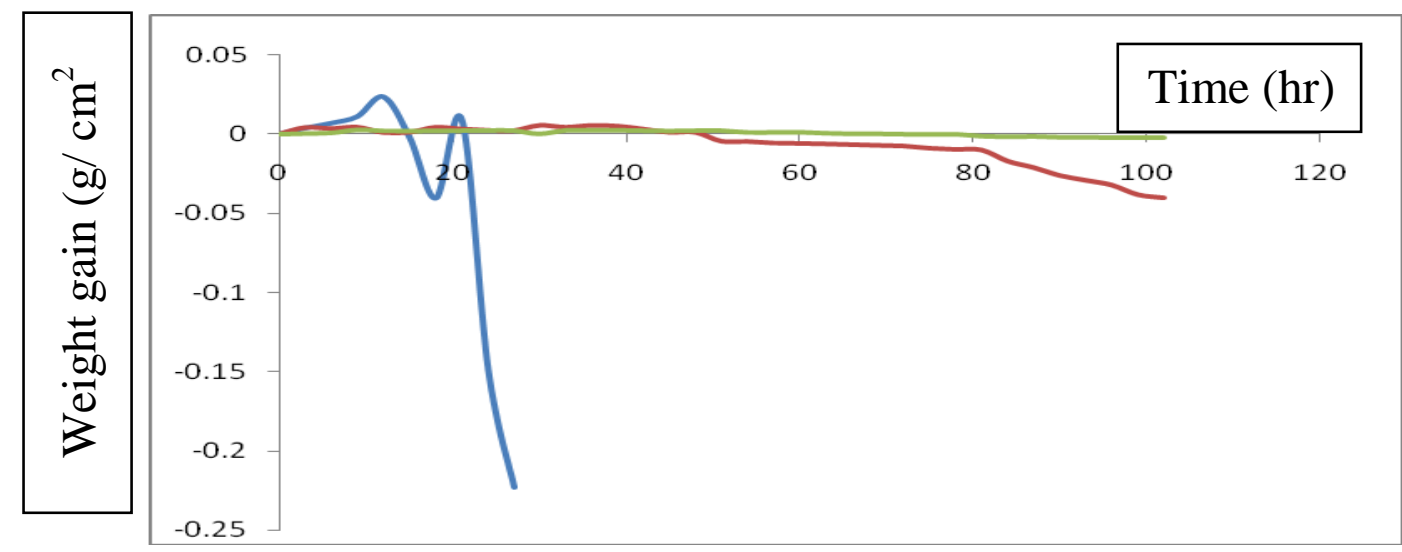

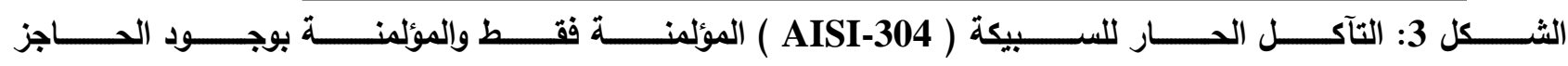
الحراري في منصهر (NaCl) بدرجة حرارة (1000 (100).

الجدول 4: الأطوار المتكونة نتيجة الأكسدة الدورية في منصهر كلوريد الصوديوم

\begin{tabular}{|c|c|c|c|c|c|c|}
\hline & \multicolumn{6}{|c|}{ الأطوار المتكونة نتيجة الاكسدة الدورية في منصهر كلوريد الصديوم بنسبة 100\% (100\%) (100) } \\
\hline $\mathrm{NiCr}_{2} \mathrm{O}_{4}$ & $\mathrm{FeCr}_{2} \mathrm{O}_{4}$ & $\mathrm{Cr}_{3} \mathrm{O}_{4}$ & $\mathrm{Fe}_{3} \mathrm{O}_{4}$ & $(\mathrm{Fe}, \mathrm{Cr})_{2} \mathrm{O}_{3}$ & $\mathrm{Fe}_{2} \mathrm{O}_{3}$ & الأطوار \\
\hline محتمل & ضعيف & قوي & ضعيف & وسط & قوي & الثدة \\
\hline
\end{tabular}

الالمنه بوجود الحاجز الحراري اوكسيد الاسبيرسيوم إن التركب ألطلائي لا يختلف كثيرا عما هو عليه في طيلاء الألمنيوم المنفرد باستثناء الفرق في السمك وظهور أطوار لعy العنصر اثبـ الفحص بالأثـعة السينية الثـكل (4) وجود الطـور الأساسـي لطبقة الطـلاء ( FeAl)، وربمـا وجـود الطور الغني بالألمنيوم (

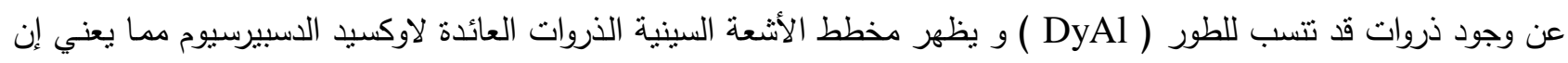
هذا الاوكسيد قد تحلل جزئيا خلال عملية الألمنة إلى ايوناته وانتشار ايونات الدسبيرسيوم إلى داخل طبقة الطلاء واتحاده بالألمنيوم

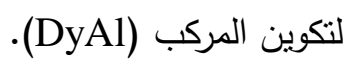

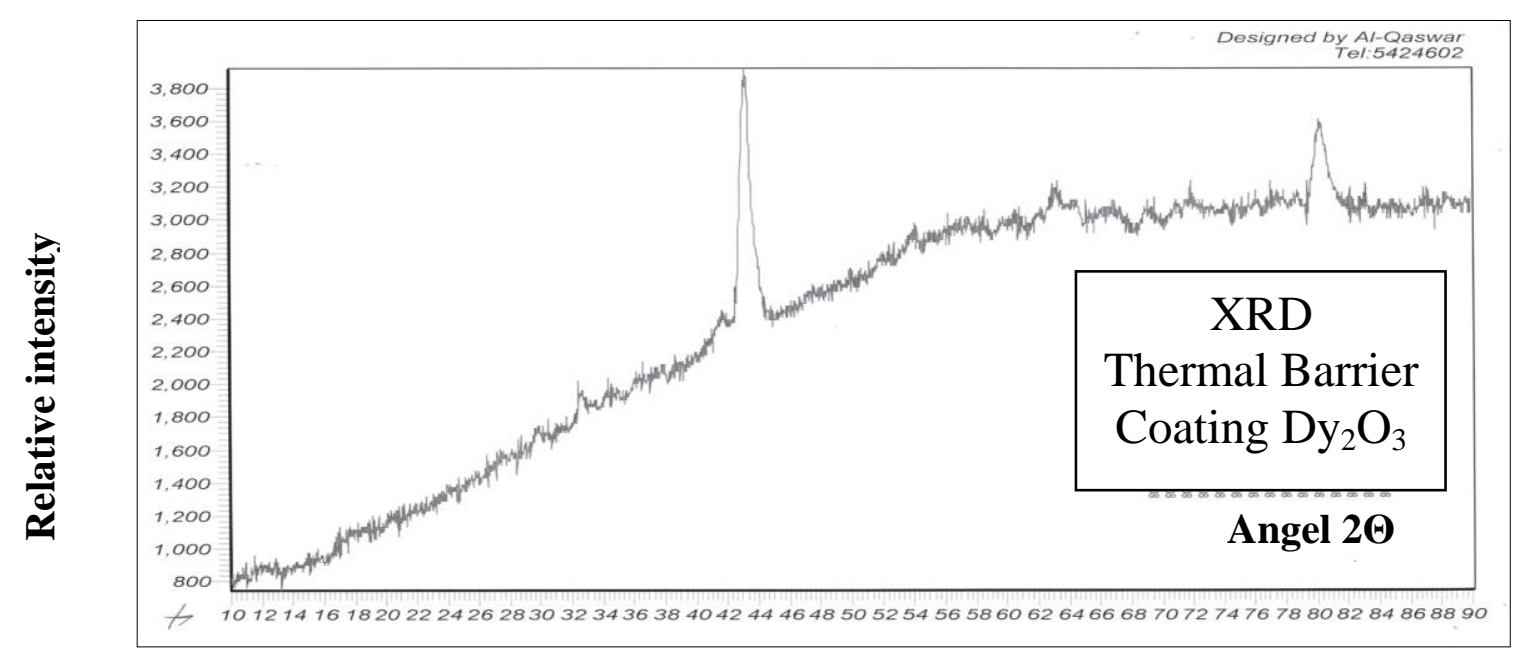

الشـكل 4: مخطط حيـود الأثـعة السـينية للنمـاذج المؤلمنـة لمـدة سـاعتين بوجـود الحساجز الحـراري اوكسيد الدسبيرسـيوم .$\left(\mathrm{Dy}_{2} \mathrm{O}_{3}\right)$ 
أكسدة سبيكة الصلب الأوستينايتي (AISI-304) المؤلمنه بوجود الحاجز الحراري اوكسيد الاسبيرسيوم (Dy2

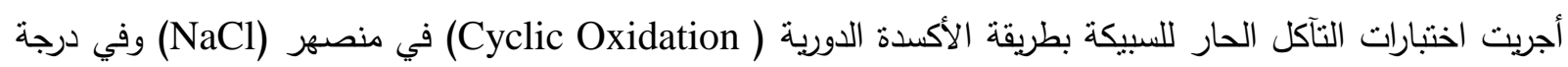

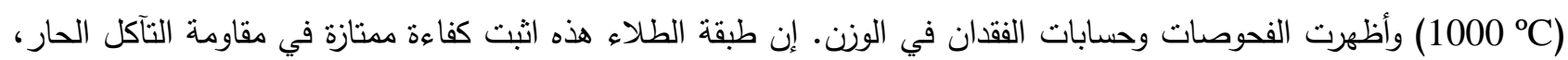

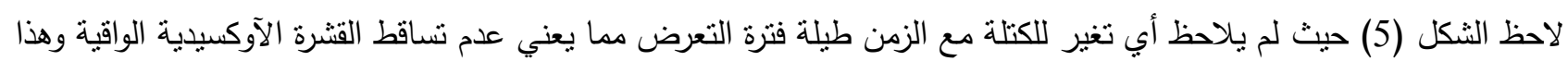
يدل على إن هذه القشرة تتمتع بالتصاقية جيدة.

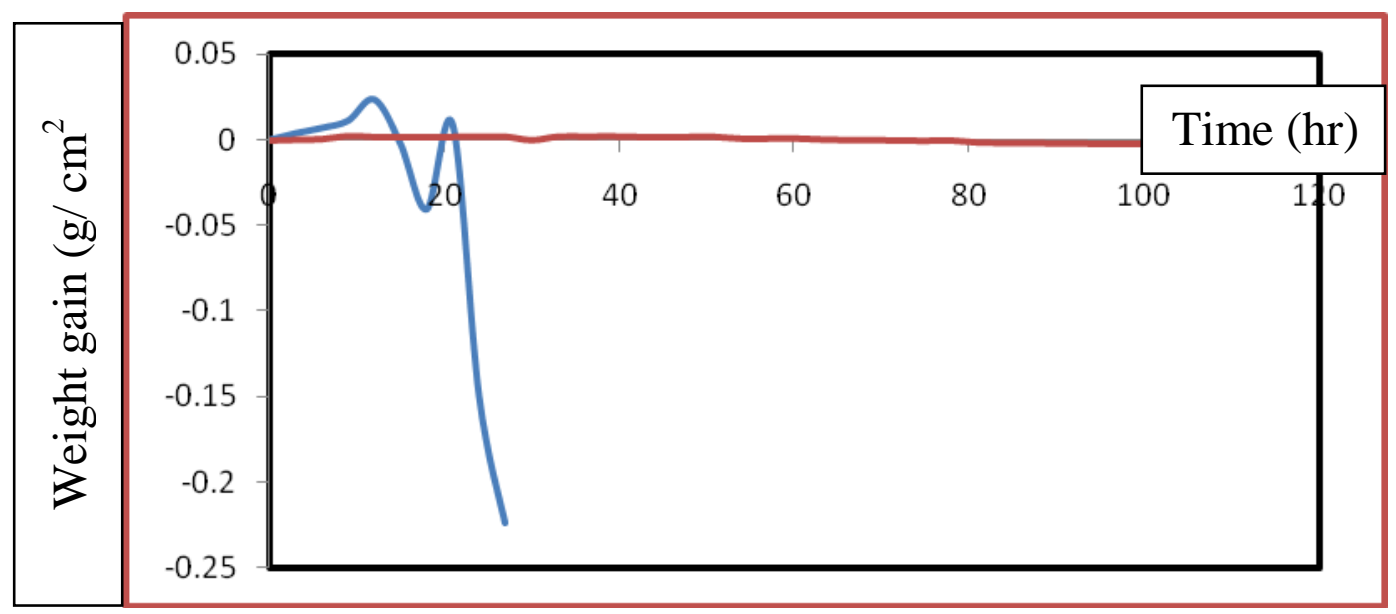

الثكل 5: الأكسدة الدورية للسبيكة (AISI-321) المؤلمنـة بوجود الحاجز الحراري (Dy2 (DaCl) في منصهر (Na) بلرجة حرارة (1000

إن طبقة اوكسيد الألمنيوم

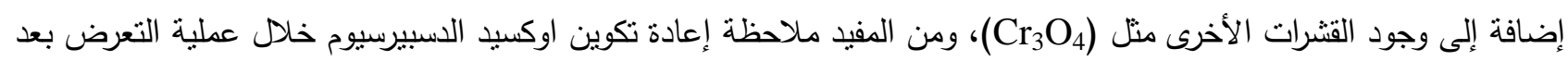
إن كان قد اختفى خلال عملية الألمنه. وهذا يعني عدم تكون مركبات كلوريدية والتي تعتبر المسببات الرئيسية لفقدان التصاقية القشرة الواقية وتساقطها. ومن هذا يبدو إن اوكسيد الدسبيرسيوم يلعب دورا مهما في نوفير الحماية كونه يعتبر من الحواجز الحرارية

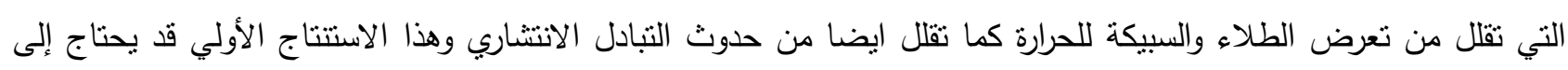
دراسة معقة للوصول إلى الحقائق الأخرى التي قد يؤديها هذا الأوكسيد.

\section{الاستنتاجات و المناقشة}

أن معدلات الوزن المكتسب وسمك الطـلاء الناتج تزداد مـع الزمن وفقاً لعلاقة القطع المكافئ لكلا الطلائين المنفرد

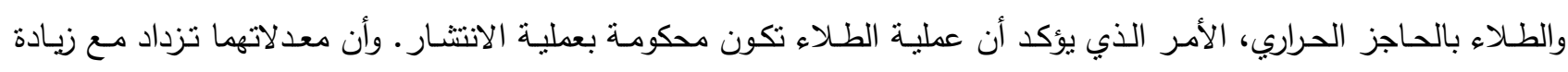
درجات الحرارة.

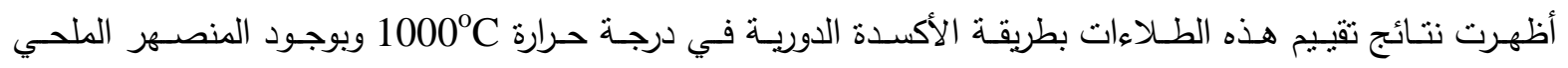
(NaCl) أنّ الطلاء بالحاجز الحراري قد وفرّ حماية ممتازة للسبيكة طوال مدّة التعرض (120) ساعة ويعزى ذلك إلى إلى التحسن

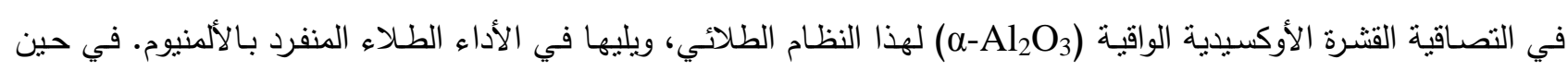

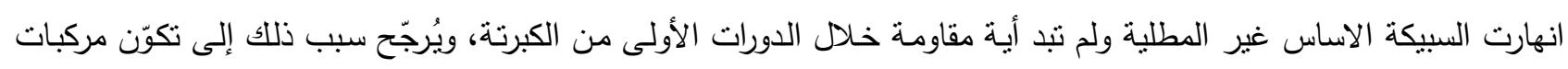

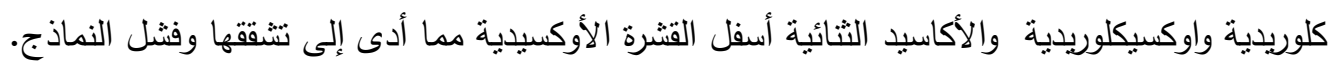



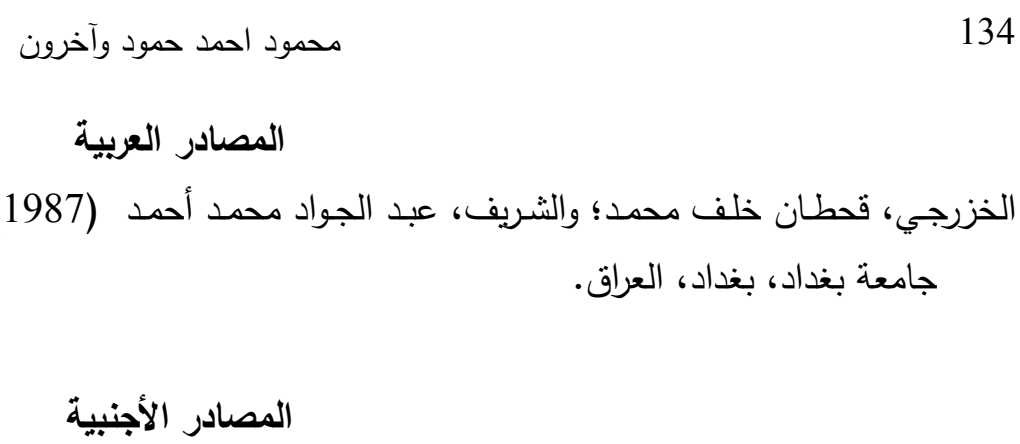

Bayer, G.T.; Wynns, K.A. (1996). Aluminide steel for sulphidation corrosion resistance. PTQ.

Chen, M.W.; Glynn, M.L.; Ott, R.T.; Hufnagel; Hemker, K.J. (2003). "Characterization and modeling of a martensitic transformation in a platinum modified diffusion aluminide bond coat for thermal barrier coatings". Acta. Materialia, 51, 279-294.

Higginson, R.L.; Jeepson, M.A.E.; West, G.D. (2006). Materials science and technology. 22(11), 1325.

Hounginou, C.; Chevalier, S.; Larpin, J.P. (2003). Aluminde coatings on stainless steels by pack cementation : high temperature reactivity. Ann. Chem. Sci. Mat., 175-184.

Hounginou, C.; Chevalier, S.; Larpin, J.P. (2003). High temperature oxidation of aluminide coatings obtained by pack cementation. Ann. Chem. Sci. Mat.

Khana, A.S. (2004). Introduction to high temperature oxidation and corrosion. ASM International.

Korinko, P.S.; Scogin, J.H.; Clark, E.A. (2001). "Development of Aluminide Coatings for Hydrogen Isotope Permeation Resistance". U.S. Department of Energy.

Levin, L.; Ginzburg, A.; Klinger, K.; Werber, T.; Kata/sman, A.; Schaat, P. (1998). Controlled formation of surface layer by pack aluminization. Surface and Coating Technology, 106, 209-213.

Morsi, K. (2001). Review : reaction synthesis processing of Ni-Al intermetalic materials. Materials Sci. and Engin., 299A, 1-15.

Mudali, U.K.; Bhuvaneswaran, N.; Shankar, P.; Khatak, H.S.; Raj, B. (2004). Intermetallic diffusion coating for enhanced hot-salt oxidation resistance of nitrogen-coating austenitic stainless steels. Metallurgical and Material Transactions A, 33A.

Seifedine, K. (2008). Corrosion Analysis of stainless steel. European J. Scientific Research, ISSN 1450- 216X, 22(4), 508-516.

Shahrour, K.M.N. (1994). Some aspects of chromium and silicon-aluminide on 321 stainless steel alloy. Thesis submitted to University of Mosul, College of Science.

Squillace, A.; Bonettie, R.; Archer, N.J. (1999). The control of the composition and structure of aluminide layers formed by vapor aluminizing. Surface and Coating Technology, 153, 120-123.

Tatsia, W.; Enhuang, K. (2000). Microstructure aspect and oxidation resistance of an aluminide coating on 310 stainless steel. Thin Solid Films, 164-168.

Wang, C.J.; He, T.T. (2002). Oxid. Met. 58, 415. 